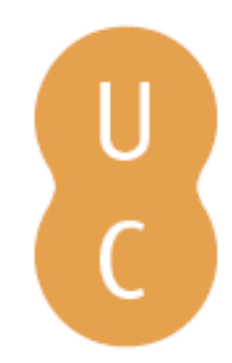

\title{
nommalina
}

\section{Forest fires hotspots in EU Southern Member States and North Africa: a review of causes and motives}

Autor(es): $\quad$ Tedim, F.; Meddour-Sahar, O.; Lovreglio, R.; Leone, V.

Publicado por: Imprensa da Universidade de Coimbra

URL

persistente: URI:http://hdl.handle.net/10316.2/34052

DOI: $\quad$ DOI:http://dx.doi.org/10.14195/978-989-26-0884-6_205

Accessed : $\quad$ 6-Feb-2015 15:00:43

A navegação consulta e descarregamento dos títulos inseridos nas Bibliotecas Digitais UC Digitalis, UC Pombalina e UC Impactum, pressupõem a aceitação plena e sem reservas dos Termos e Condições de Uso destas Bibliotecas Digitais, disponíveis em https://digitalis.uc.pt/pt-pt/termos.

Conforme exposto nos referidos Termos e Condições de Uso, o descarregamento de títulos de acesso restrito requer uma licença válida de autorização devendo o utilizador aceder ao(s) documento(s) a partir de um endereço de IP da instituição detentora da supramencionada licença.

Ao utilizador é apenas permitido o descarregamento para uso pessoal, pelo que o emprego do(s) título(s) descarregado(s) para outro fim, designadamente comercial, carece de autorização do respetivo autor ou editor da obra.

Na medida em que todas as obras da UC Digitalis se encontram protegidas pelo Código do Direito de Autor e Direitos Conexos e demais legislação aplicável, toda a cópia, parcial ou total, deste documento, nos casos em que é legalmente admitida, deverá conter ou fazer-se acompanhar por este aviso.

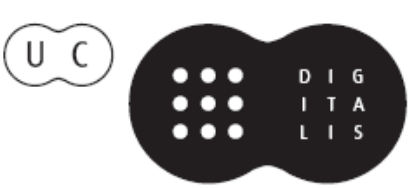




\section{ADVANCES IN}

Forest Fire

\section{RESEARCH}

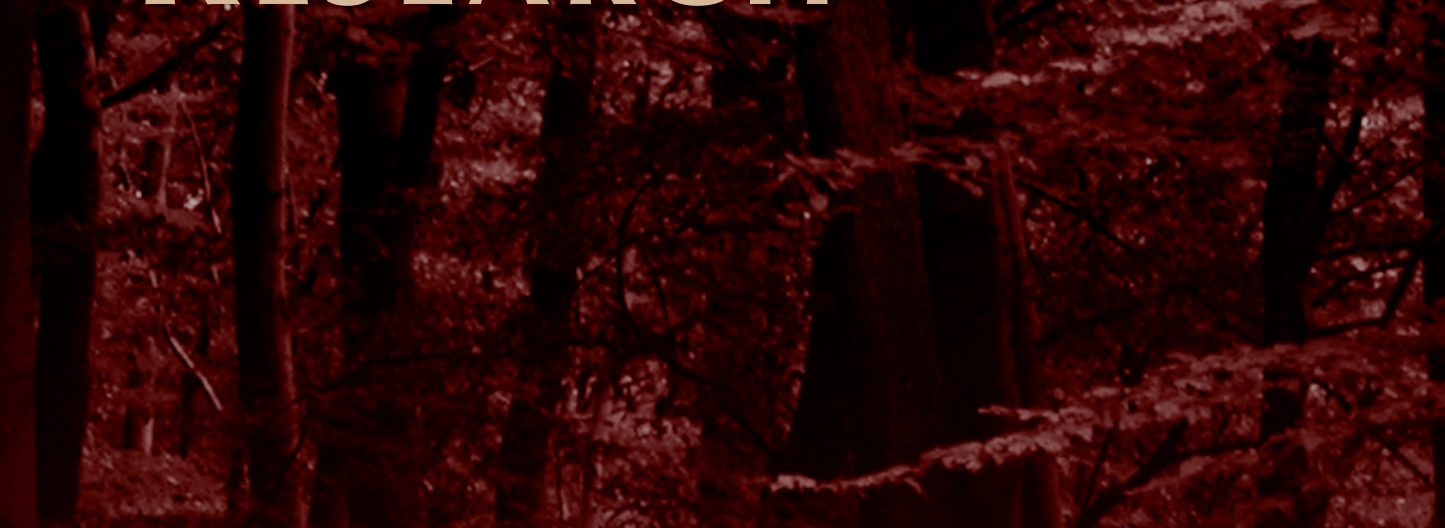

\section{DOMINGOS XAVIER VIEGAS}

\section{EDITOR}




\title{
Forest fires hotspots in EU Southern Member States and North Africa: a review of causes and motives
}

\author{
Tedim F. ${ }^{\text {, }}$ Meddour-Sahar O. ${ }^{\mathrm{b}}$, Lovreglio R. ${ }^{\mathrm{c}}$, LeoneV. ${ }^{\mathrm{d}}$ \\ ${ }^{a}$ University of Porto, Faculty of Arts, Via Panorâmica s/n 4150-564 Porto, Portugal, \\ ftedim@letras.up.pt \\ ${ }^{b}$ University Mouloud Mammeri, Faculty of Biological Sciences and Agronomy, BP 17 RP, Tizi \\ Ouzou, 15000,Algeria, o.sahar@yahoo.fr \\ ${ }^{c}$ University of Sassari, Department of Agriculture, v. Cristoforo Colombo 1, I-08100 Nuoro, Italy, \\ rlovreglio@uniss.it \\ ${ }^{d}$ University of Basilicata (retired), Department of Crop Systems, Forestry and Environmental \\ Sciences, I-85100 Potenza, Italy, vittorio.leone@tiscali.it
}

\begin{abstract}
Despite enhanced fire suppression efforts the number of fires has increased in Europe and in North Africa, above all in Portugal, and the extinction oriented model of fire control is no more suitable to change the trend. Hence, the need of shifting from a reactive model grounded on a fast and strong reaction, to more pro-active procedures supported by prevention, aimed not only to fuel reduction but also to gradually intervene on the causes of fires. In this perspective, a fair knowledge of man-caused fire outbreak motives is a crucial point. This paper intended to compare and interpret those motives in Portugal, Italy and Algeria obtained through the perception of experts (forest experts and other practitioners). In this research new data was collected in the North Region of Portugal and data already available for Algeria and Italy was commented.

The results of the research highlighted great similarities of the main fire causes and motives identified by the experts from the case-studies in the three countries. Fire outbreak motives are not related with deliberate fire setting but, mainly, with the use of fire in rural activities. Fire is a manageable tool that is still needed in many agro-forestry systems. One of the findings of this study pointed out the need to design and develop specific prevention programs able to reduce fire incidence and promote practices more respectful of the tradition and needs of rural communities, often criminalized for their habitudes of using fire as a multipurpose tool. Deliberate fire can represent isolated and sporadic actions however, some of them result from organized and even criminal interests. Finally, this paper argues that improved fire prevention to address the roots of fire problems cannot be achieved without a deep knowledge of the complexity and diversity of the fire outbreak motives.
\end{abstract}

Keywords: CBFiM, concertation, Delphi technique, EU, fire outbreaks motives, forest fire cause, integrated fire management, North Africa, occupational fire, prevention

\section{Introduction}

Each year burned area continues to be substantial in Europe and in other Mediterranean countries (Table 1). Enhanced fire suppression efforts and investments in human and technical resources (e.g. important aerial fleets; updated methods and technology to fight fires; emergency response organization, coordination and support) limited the damages but did not solve the fire problem which is far from being controlled. The incapacity of reducing the number of ignitions, the apparition of events that grow to previously unheard sizes, and more frequent and complex fires in the wildland urban interface, are alerting signals of the need to improve and change forest fires policies. A mainly extinction oriented model of fire control seems not well adapted to cope with new forest fire scenarios (Molina et al. 2010; Montiel and Herrero 2010; Sebastián López et al. 2011). 
Table1 - Forest fires in Southern European and North Africa countries

\begin{tabular}{|c|c|c|c|c|c|}
\hline Country & $\begin{array}{c}\text { Average yearly } \\
\text { burnt area (ha) }\end{array}$ & $\begin{array}{c}\text { Average yearly } \\
\text { number of fires } \\
\text { (no.) }\end{array}$ & $\begin{array}{c}\text { Maximum } \\
\text { annual burnt } \\
\text { area (ha) }\end{array}$ & $\begin{array}{c}\text { Average forest } \\
\text { fire density } \\
\text { no. fires/ km }\end{array}$ & AAR $^{* * *}$ \\
\hline Spain (1980-2012) & 171,593 & 15,263 & $484,476^{1}$ & 3.0 & 0.84 \\
\hline Portugal (1980-2012) & 108,334 & 18,613 & $425,726^{2}$ & 20.3 & 3.31 \\
\hline Italy (1980-2012) & 113,496 & 9,736 & $227,729^{3}$ & 3.2 & 1.14 \\
\hline Greece (1980-2012) & 47,141 & 1,554 & $225,734^{3}$ & 1.2 & 0.57 \\
\hline Algeria (1986- 2010) & 35,025 & 1,637 & $271,598^{4}$ & 0.07 & 0.85 \\
\hline France (1980-2012) & 26,383 & 4,880 & $75,566^{5}$ & 0.9 & 0.53 \\
\hline Morocco (1960-2009) & 2,987 & 260 & $11,174^{6}$ & 0.06 & $<0.01$ \\
\hline Tunisia (2005-2010) & 1,554 & 750 & $3,551^{7}$ & 0.5 & $<0.01$ \\
\hline
\end{tabular}

${ }^{1}$ In $1985 ;{ }^{2}$ In $2003 ;{ }^{3}$ In $2007 ;{ }^{4}$ In $1994 ;{ }^{5}$ In $1989 ;{ }^{6}$ In $2012 ;{ }^{7}$ In 2010

Source: *European Commission, 2013; ${ }^{* *}$ Annual Average Risk (AAR) i.e. the percentage of the wooded area burnt each year (FAO, 1999)

Even though forest fires have not usually been considered as a natural hazard by the scientific community (McCaffrey 2004), since the last decades of 20th century they have been recognized as natural hazards (e.g. floods, earthquakes) by the state agencies and institutions, such as NASA or European Union (EU) (Leone and Lovreglio 2003) and, consequently, as a problem to be covered by Civil Protection (or Civil Defence in some countries). Forest fires are a civil protection/defence problem in the moment of suppression, but they are the result of complex interrelationships between society-environment-economy, thus requiring a rather different approach shifting from the reactive model, typical of Civil Protection, to more pro-active procedures supported by a prevention strategy (EFIMED 2012; Xanthopoulos 2012; Marino et al. 2014; Raftoyannis et al. 2014).

A symptomatic solution dealing with problems, as they arise, but failing to address the underlying causes, can increase the chance that the same problem will crop up in the future. In the case of forest fires, this means to allocate resources to short-term fixing of symptoms, increasing emphasis on fire suppression, instead of dealing with the causes of problems (Collins et al. 2013), so neglecting fire prevention. The "firefighting trap" metaphor as used in business domain (Collins 2012; Collins et al. 2013) well depicts such a short sighted cycle of problem-solving.

Policy makers and fire managers should pursue a balanced approach to suppression and prevention activities (Collins et al. 2013) aimed not only at fuel management but also to intervene on the causes of forest fires which are predominantly the result of anthropic activities (FAO 2012).Thus, actions must effort to change individual, social and institutional behaviours, but they meet a weak point in the general lack of in-depth knowledge of causes and forest fire outbreaks motives which according to FAO (1999) is a "precondition for the implementation of prevention adapted solutions" (p. 41). It is actually biased by the relevant incidence of unknown causes [e.g. 95\% in Morocco (European Commission 2013), 80\% in Algeria (Meddour-Sahar et al. 2013), 60\% in Tunisia (Ben Jamaa and Belhaj 2011), 16\% in Italy (CFS 2012), 49\% in Portugal (ICNF 2012)]. This is due to the current system of producing forest fires statistics through an a-critical processing of individual forest fires reports, compiled soon after control activity is over. The task is difficult without appropriate surveys, which are impossible in the presence of a great number of events, most of them of less than 5 ha, and without the identification of each ignition point.

This study intended to interpret the motives (i.e. "the intentional state which prompts or moves one to act"; Wrathall 2005: p. 118) of forest fires outbreaks in Portugal through the perception of forest experts, and compared the results with previous studies realized in Italy and Algeria, by a method which consistently reduces the incidence of unknown motives. This paper argues that improved fire 
prevention to address the roots of fire problems requires a deep knowledge on the complexity and diversity of man-induced fire causes and motives and should be sensitive to their spatial variation.

\section{Methods}

In the absence of sufficient data and/or complete knowledge in regard to forest fires motives the Delphi technique, grounded on a structured process to collect opinions from a group of experts, can be used. This collective judgment, although made up of subjective opinions, is considered to be more reliable than individual statements and is thus more objective in its outcomes.

In Portugal, a research of forest fires causes using Delphi method was conducted in the North Region (NUT 2), with about 3.6 millions of inhabitants (INE 2012) and a surface of $21,278 \mathrm{~km}^{2}$. It is among the most fire prone regions of the country where, between 1980 and 2011, a total number of 351,550 fires was recorded which burned 1,219,609 ha. The North Region encompasses 86 municipalities, but the urban municipalities of Porto and S. João da Madeira were excluded from the analysis. To the 84 selected municipalities a questionnaire was sent by email to be answered by the Forest Fire Technical Division of each municipality which is responsible for the Municipal Plan for the Defence of the Forest Against Fire, as established by the Decree-law 17/2009. The questionnaire contained the Portuguese official list of the three level structure of fire causes reporting 68 motives (http://www.icnf.pt/portal/florestas/dfci/Resource/doc/estatist/dgrf-codificacao-causas.pdf; Camia et al. 2013), divided into seven main categories (i.e. negligent usage of fire, accidental, structural causes, incendiary, natural, unknown, and rekindle) with their identification codes. Experts should identify the fire outbreaks motives in their territories, classifying them by a Likert scale from 1 to 5 (ranging from not important to extremely important); successively, they were asked to rank the six most important motives in decreasing order of importance from 1 (the most important) to 6 (the least important). Sixty nine valid answers were received (i.e. a return rate of $82 \%$ of the contacted sample). The information from the questionnaire was analysed and a fire motive rank-ordering was established as the mode of rank-scores. The early results of this procedure are presented and discussed in this paper. The following step will be the organization of meetings in different municipalities of the North Region to circulate, validate and discuss the results from the questionnaire.

The procedure used in Portugal was slightly different from the one used in Algeria and Italy concerning the two rounds interspersed with a feedback and the number of ranked motives, only six in Portugal instead of the eight in Algeria and Italy. These differences did not have implications in the comparative study. Detailed information on the methodology used in Algeria and Italy can be found in MeddourSahar et al. (2013) and Lovreglio et al. (2006), respectively.

In Algeria, the Delphi method was used to identify forest fires causes and motives in the three most affected wilayas (equivalent of NUTS 3, Departments) i.e. Bouira, Boumerdes, and Tizi Ouzou (Meddour-Sahar et al. 2013; Meddour-Sahar 2014).

In Italy, the same method was used in the 29 most affected provinces in the Centre and South of the country, in two National Parks (NP) in the South of the Country (NP of Gargano, NP of Cilento and Vallo di Diano) and in some minor administrative units such as Mountain Communities (MC) (Lovreglio et al. 2006; De las Heras et al. 2007; Lovreglio et al. 2008; Leone and Lovreglio 2009; Lovreglio et al. 2010a,b; Marciano et al. 2010; Regione Puglia 2012).

Although it was interesting to consider Spain in this study, the data obtained also using Delphi method (APAS and IDEM 2003, 2004; Dolz Reus and Franco Irastorza 2005; Franco Irastorza and Dolz Reus 2007) were not comparable for methodological differences.

\section{Results}

The frequency analysis of forest fires outbreak motives reported as extremely important by the panel's members in North Region of Portugal showed a large variety of motives, where just five of 68 possible reasons were not identified as extremely important by the experts. The most mentioned were rekindle 
(considered by $54 \%$ of the municipalities) and management of forestry or agricultural vegetation (e.g. renewing pastures, $48 \%$ of the municipalities' responses; clearing of forest areas, $45 \%$; clearing of agricultural areas, $41 \%$ ). Other motives were related with conflicts in the use of resources (e.g. hunting conflicts, 30\%), influence on economic/market activities (e.g. pressure on wood market, 20\%), social and interpersonal tensions (e.g. conflicts between neighbours, 26\%; revenges and retaliatory actions, $24 \%$ ), and antisocial behaviours (e.g. pyromania, 38\%; vandalism, 32\%).

The rank obtained from the modal values of the extremely important motives classified from 1 to 6 by the panel's members highlighted the same reasons although presenting them in slightly different order (Table 2). Thus, the most important fire ignition motive in the North Region of Portugal was renewing pastures which was identified as the first one in 16 municipalities and the second one in another nine municipalities. Still related with management of forestry or agricultural vegetation cleaning the agricultural lands and cleaning forest lands appeared in rank 2 and 3, respectively. Pyromania appeared in rank 4, being the main motivation in eight municipalities. In rank 5, appeared vandalism as well as rekindle. Conflicts between neighbours were classified in rank 6.

The results of the rank ordering in North Region of Portugal were not very different from the ones found in Algeria and Italy. Furthermore, in these countries fire, as a manageable tool needed in many agro-forestry systems, appeared as the main reason for fires outbreaks. In Algeria (Table 3), the use of fire in agricultural works was the main motive in the three wilayas together with vegetation management activities (i.e. creation or renewal of pastures, forest works, and wild honey hunting). Illegal garbage dumping and burning, mentioned in the three wilayas although in different rank position, is an 'inevitable 'problem solving' solution by inhabitants, who have no other more sustainable alternative for waste disposal" (Meddour-Sahar et al. 2013: p. 251). Other negligent reason was cigarettes remains. Deliberate fires (i.e. intentionally caused by human, according to the harmonized classification scheme of fire causes in EU, Camia et al. 2013) were mainly related with interests in land use changes. Rekindle appeared important in Algeria too, where it is caused by fire crews who do not always ensure the mopping up of controlled fires but as well as by the so called "security fires" (Meddour-Sahar et al. 2013).

In Italy (Table 4), with the exception of the Province of Bari, the negligent motives are also more important than deliberate use of fire. Multiple motives in the same rank order reflect the

Table2 -Experts panel perception on forest fire ignitions motivations, in Portugal (answers N=69; orange colour for deliberate causes, green for negligent causes, and grey for rekindle)

\begin{tabular}{|c|c|c|c|c|c|c|c|c|}
\hline Frequency & Rank & \multirow{5}{*}{ Motives } & \multicolumn{5}{|c|}{ Modal values for its position category } \\
\cline { 4 - 8 } & & & $1^{\text {st }}$ & $2^{\text {nd }}$ & $3^{\text {rd }}$ & $4^{\text {th }}$ & $5^{\text {th }}$ & $6^{\text {th }}$ \\
\hline 33 & 1 & Renewing pastures & 16 & 9 & 1 & 0 & 1 & 1 \\
\hline 28 & 2 & Cleaning of agricultural areas & 1 & 10 & 3 & 3 & 4 & 0 \\
\hline 31 & 3 & Cleaning of forest areas & 3 & 3 & 9 & 5 & 2 & 3 \\
\hline 26 & 4 & Pyromania & 8 & 3 & 3 & 7 & 1 & 2 \\
\hline 22 & 5 & Vandalism & 8 & 2 & 1 & 2 & 3 & 0 \\
\hline 37 & 2 & Rekindle & 8 & 6 & 6 & 2 & 3 & 2 \\
\hline 18 & 6 & Conflicts between neighbours & 1 & 4 & 4 & 0 & 2 & 4 \\
\hline
\end{tabular}


Table 3. Perception of experts' panels on forest fire ignitions motivations in Algeria (orange colour for deliberate causes, green for negligent causes, and rekindle in grey)

\begin{tabular}{|c|c|c|c|c|c|c|c|}
\hline Wilaya & 1 & 2 & 3 & 4 & 5 & 6 & Source \\
\hline Bouira & $\begin{array}{c}\text { Agricultural } \\
\text { works } \\
\text { (stubble } \\
\text { burning) }\end{array}$ & $\begin{array}{l}\text { Cigarette } \\
\text { remains }\end{array}$ & $\begin{array}{c}\text { Illegal } \\
\text { garbage } \\
\text { dumping and } \\
\text { burning }\end{array}$ & $\begin{array}{l}\text { Creation or } \\
\text { renewal of } \\
\text { pastures }\end{array}$ & $\begin{array}{l}\text { Interests in } \\
\text { land use } \\
\text { changes }\end{array}$ & $\begin{array}{l}\text { Wild honey } \\
\text { hunting }\end{array}$ & $\begin{array}{l}\text { Meddour } \\
\text {-Sahar et } \\
\text { al. } 2013\end{array}$ \\
\hline $\begin{array}{c}\text { Boumer } \\
\text { des }\end{array}$ & $\begin{array}{c}\text { Agricultural } \\
\text { works } \\
\text { (stubble } \\
\text { burning) }\end{array}$ & Rekindle & $\begin{array}{l}\text { Forest works } \\
\text { (burning of } \\
\text { cut bush) }\end{array}$ & $\begin{array}{c}\text { Illegal } \\
\text { garbage } \\
\text { dumping and } \\
\text { burning }\end{array}$ & $\begin{array}{l}\text { Cigarette } \\
\text { remains }\end{array}$ & $\begin{array}{l}\text { Interests in } \\
\text { land use } \\
\text { changes }\end{array}$ & \multirow[t]{2}{*}{$\begin{array}{c}\text { Meddour } \\
\text {-Sahar } \\
2014\end{array}$} \\
\hline $\begin{array}{l}\text { Tizi- } \\
\text { Ouzou }\end{array}$ & $\begin{array}{c}\text { Agricultural } \\
\text { works } \\
\text { (stubble } \\
\text { burning) }\end{array}$ & $\begin{array}{c}\text { Illegal } \\
\text { garbage } \\
\text { dumping and } \\
\text { burning }\end{array}$ & $\begin{array}{c}\text { Interests in } \\
\text { land use } \\
\text { changes }\end{array}$ & $\begin{array}{c}\text { Cigarette } \\
\text { remains }\end{array}$ & Rekindle & $\begin{array}{l}\text { Forest works } \\
\text { (burning of } \\
\text { cut bush) }\end{array}$ & \\
\hline
\end{tabular}

Table4 -Perception of experts' panels on forest fire ignitions motivations in Italy (N.A.-Not available; orange colour for deliberate causes, green for negligent causes, and rekindle in grey)

\begin{tabular}{|c|c|c|c|c|c|c|c|}
\hline NUTS & 1 & 2 & 3 & 4 & 5 & 6 & Source \\
\hline $\begin{array}{l}\text { NUTS2 } \\
\text { Basilicat } \\
\text { a Region }\end{array}$ & $\begin{array}{l}\text { Agricultura } \\
1 \text { works } \\
\text { (stubble } \\
\text { burning) }\end{array}$ & $\begin{array}{l}\text { Creation or } \\
\text { renewal of } \\
\text { pastures }\end{array}$ & $\begin{array}{c}\text { Agricultural } \\
\text { works } \\
\text { (stubble } \\
\text { burning) }\end{array}$ & $\begin{array}{l}\text { Creation or } \\
\text { renewal of } \\
\text { pastures }\end{array}$ & $\begin{array}{c}\text { Ownership } \\
\text { controversie } \\
\text { s }\end{array}$ & $\begin{array}{c}\text { Retaliation } \\
\text { against public } \\
\text { administratio } \\
n\end{array}$ & $\begin{array}{c}\text { Lovregli } \\
\text { o et al. } \\
2006\end{array}$ \\
\hline $\begin{array}{c}\text { NUTS3 } \\
\text { Province } \\
\text { of } \\
\text { Taranto }\end{array}$ & $\begin{array}{l}\text { Burning of } \\
\text { fallow land }\end{array}$ & $\begin{array}{c}\text { Agricultural } \\
\text { works } \\
\text { (stubble } \\
\text { burning) }\end{array}$ & $\begin{array}{c}\text { Agricultural } \\
\text { works } \\
\text { (stubble } \\
\text { burning) }\end{array}$ & $\begin{array}{l}\text { Creation or } \\
\text { renewal of } \\
\text { pastures }\end{array}$ & $\begin{array}{l}\text { Creation or } \\
\text { renewal of } \\
\text { pastures }\end{array}$ & $\begin{array}{c}\text { Illegal } \\
\text { garbage } \\
\text { dumping and } \\
\text { burning }\end{array}$ & $\begin{array}{c}\text { Lovregli } \\
\text { o et al. } \\
2008\end{array}$ \\
\hline \multirow[t]{3}{*}{$\begin{array}{c}\text { MC of } \\
\text { Vallo di } \\
\text { Diano }\end{array}$} & $\begin{array}{l}\text { Agricultura } \\
\text { l uses }\end{array}$ & \multirow[t]{3}{*}{$\begin{array}{l}\text { Agricultural } \\
\text { works } \\
\text { (stubble } \\
\text { burning) }\end{array}$} & $\begin{array}{c}\text { Hunting } \\
\text { products in } \\
\text { areas } \\
\text { scorched by } \\
\text { fire passage } \\
\text { (e.g. } \\
\text { mushrooms, } \\
\text { wild } \\
\text { asparagus) } \\
\end{array}$ & $\begin{array}{l}\text { Cigarettes } \\
\text { remains }\end{array}$ & $\begin{array}{c}\text { Hunting } \\
\text { products in } \\
\text { areas } \\
\text { scorched by } \\
\text { fire passage } \\
\text { (e.g. } \\
\text { mushrooms, } \\
\text { wild } \\
\text { asparagus) }\end{array}$ & \multirow[t]{3}{*}{$\begin{array}{c}\text { Fire-crackers } \\
\text { and bottle- } \\
\text { rockets }\end{array}$} & \multirow[t]{3}{*}{$\begin{array}{c}\text { Lovregli } \\
\text { o et al. } \\
2010 \mathrm{a}\end{array}$} \\
\hline & \multirow[t]{2}{*}{$\begin{array}{l}\text { Burning of } \\
\text { rests }\end{array}$} & & $\begin{array}{c}\text { Agricultural } \\
\text { works } \\
\text { (stubble } \\
\text { burning) }\end{array}$ & \multirow[t]{2}{*}{$\begin{array}{l}\text { Agricultural } \\
\text { uses }\end{array}$} & $\begin{array}{l}\text { Burning of } \\
\text { rests }\end{array}$ & & \\
\hline & & & $\begin{array}{l}\text { Ownership } \\
\text { controversies }\end{array}$ & & $\begin{array}{c}\text { Ownership } \\
\text { controversie } \\
\text { s } \\
\end{array}$ & & \\
\hline \multirow[t]{3}{*}{$\begin{array}{c}\text { NP of } \\
\text { Gargano }\end{array}$} & \multirow{3}{*}{$\begin{array}{l}\text { Agricultura } \\
1 \text { works } \\
\text { (stubble } \\
\text { burning) }\end{array}$} & \multirow[t]{2}{*}{$\begin{array}{l}\text { Creation or } \\
\text { renewal of } \\
\text { pastures }\end{array}$} & $\begin{array}{l}\text { Protest of } \\
\text { seasonal fire- } \\
\text { fighters }\end{array}$ & \multirow{2}{*}{$\begin{array}{c}\text { Agricultural } \\
\text { works } \\
\text { (stubble } \\
\text { burning) }\end{array}$} & \multirow[t]{3}{*}{$\begin{array}{c}\text { Creation or } \\
\text { renewal of } \\
\text { pastures }\end{array}$} & \multirow[t]{3}{*}{$\begin{array}{l}\text { Protest of } \\
\text { seasonal fire- } \\
\text { fighters }\end{array}$} & \multirow[t]{3}{*}{$\begin{array}{c}\text { Lovregli } \\
\text { o et al. } \\
2010 \mathrm{a}\end{array}$} \\
\hline & & & $\begin{array}{c}\text { Retaliation } \\
\text { against public } \\
\text { administratio } \\
n\end{array}$ & & & & \\
\hline & & $\begin{array}{l}\text { Protest of } \\
\text { seasonal } \\
\text { fire-fighters }\end{array}$ & $\begin{array}{l}\text { Fire caused } \\
\text { with the } \\
\text { intent of } \\
\text { being } \\
\text { included in } \\
\text { fire-fighting } \\
\text { crews }\end{array}$ & $\begin{array}{l}\text { Fire caused } \\
\text { with the } \\
\text { intent of } \\
\text { being } \\
\text { included in } \\
\text { fire-fighting } \\
\text { crews }\end{array}$ & & & \\
\hline
\end{tabular}




\begin{tabular}{|c|c|c|c|c|c|c|c|}
\hline \multirow[t]{3}{*}{$\begin{array}{c}\text { NUTS3 } \\
\text { Province } \\
\text { of Bari }\end{array}$} & \multirow{2}{*}{$\begin{array}{l}\text { Fire caused } \\
\text { with the } \\
\text { intent of } \\
\text { being } \\
\text { included in } \\
\text { fire- } \\
\text { fighting } \\
\text { crews }\end{array}$} & $\begin{array}{c}\text { Agricultural } \\
\text { works } \\
\text { (stubble } \\
\text { burning) }\end{array}$ & $\begin{array}{c}\text { Agricultural } \\
\text { works } \\
\text { (stubble } \\
\text { burning) } \\
\end{array}$ & $\begin{array}{c}\text { Creation or } \\
\text { renewal of } \\
\text { pastures }\end{array}$ & $\begin{array}{l}\text { Building } \\
\text { speculation }\end{array}$ & $\begin{array}{l}\text { Hot vehicle } \\
\text { exhaust pipes }\end{array}$ & \multirow[t]{3}{*}{$\begin{array}{l}\text { Lovregli } \\
\text { o et al. } \\
2010 \mathrm{a}\end{array}$} \\
\hline & & $\begin{array}{l}\text { Protest of } \\
\text { seasonal } \\
\text { fire-fighters }\end{array}$ & $\begin{array}{l}\text { Cleaning of } \\
\mathrm{road} / \mathrm{rail} \text { road }\end{array}$ & \multirow{2}{*}{$\begin{array}{l}\text { Fire caused } \\
\text { with the } \\
\text { intent of } \\
\text { being } \\
\text { included in } \\
\text { fire-fighting } \\
\text { crews }\end{array}$} & \multirow[t]{2}{*}{$\begin{array}{c}\text { Protest of } \\
\text { seasonal } \\
\text { fire-fighters }\end{array}$} & $\begin{array}{l}\text { Building } \\
\text { speculation }\end{array}$ & \\
\hline & $\begin{array}{l}\text { Agricultura } \\
\text { 1 works } \\
\text { (stubble } \\
\text { burning) }\end{array}$ & $\begin{array}{c}\text { Ownership } \\
\text { controversie } \\
\text { s }\end{array}$ & $\begin{array}{l}\text { Protest of } \\
\text { seasonal fire- } \\
\text { fighters }\end{array}$ & & & $\begin{array}{c}\text { Ownership } \\
\text { controversies }\end{array}$ & \\
\hline \multirow{3}{*}{$\begin{array}{l}\text { NP of } \\
\text { Cilento } \\
\text { and } \\
\text { Vallo di } \\
\text { Diano }\end{array}$} & \multirow[t]{3}{*}{$\begin{array}{l}\text { Creation or } \\
\text { renewal of } \\
\text { pastures }\end{array}$} & \multirow{3}{*}{$\begin{array}{l}\text { Hunting } \\
\text { products in } \\
\text { areas } \\
\text { scorched by } \\
\text { fire passage } \\
\text { (e.g. } \\
\text { mushrooms, } \\
\text { wild } \\
\text { asparagus) }\end{array}$} & \multirow{3}{*}{$\begin{array}{c}\text { Hunting } \\
\text { products in } \\
\text { areas } \\
\text { scorched by } \\
\text { fire passage } \\
\text { (e.g. } \\
\text { mushrooms, } \\
\text { wild } \\
\text { asparagus) }\end{array}$} & $\begin{array}{c}\text { Ownership } \\
\text { Controversie } \\
\text { s }\end{array}$ & $\begin{array}{l}\text { Plantation } \\
\text { cleaning } \\
\text { after } \\
\text { harvest }\end{array}$ & \multirow{2}{*}{$\begin{array}{l}\text { Hunting } \\
\text { products in } \\
\text { areas } \\
\text { scorched by } \\
\text { fire passage } \\
\text { (e.g. } \\
\text { mushrooms, } \\
\text { wild } \\
\text { asparagus) }\end{array}$} & \multirow[t]{3}{*}{$\begin{array}{l}\text { Lovregli } \\
\text { o et al. } \\
2010 \mathrm{~b}\end{array}$} \\
\hline & & & & $\begin{array}{c}\text { Agricultural } \\
\text { land cleaning } \\
\text { after } \\
\text { harvesting }\end{array}$ & $\begin{array}{l}\text { Burning of } \\
\text { rests }\end{array}$ & & \\
\hline & & & & $\begin{array}{l}\text { Burning of } \\
\text { rests }\end{array}$ & & $\begin{array}{c}\text { Ownership } \\
\text { Controversies }\end{array}$ & \\
\hline \multirow[t]{3}{*}{$\begin{array}{c}\text { NP of } \\
\text { Gargano }\end{array}$} & \multirow[t]{3}{*}{$\begin{array}{l}\text { Creation or } \\
\text { renewal of } \\
\text { pastures }\end{array}$} & \multirow[t]{3}{*}{$\begin{array}{l}\text { Agricultural } \\
\text { works } \\
\text { (stubble } \\
\text { burning) }\end{array}$} & \multirow{3}{*}{$\begin{array}{l}\text { Vegetation } \\
\text { burning to } \\
\text { earn } \\
\text { agricultural } \\
\quad \text { land }\end{array}$} & \multirow[t]{3}{*}{$\begin{array}{l}\text { Burning of } \\
\text { fallow land }\end{array}$} & \multirow[t]{3}{*}{$\begin{array}{l}\text { Burning of } \\
\text { rests }\end{array}$} & $\begin{array}{c}\text { Agricultural } \\
\text { works } \\
\text { (stubble } \\
\text { burning) }\end{array}$ & \multirow[t]{3}{*}{$\begin{array}{l}\text { Leone } \\
\text { and } \\
\text { Lovregli } \\
\text { o } 2009\end{array}$} \\
\hline & & & & & & $\begin{array}{c}\text { Fire lit to } \\
\text { depreciate } \\
\text { tourist areas }\end{array}$ & \\
\hline & & & & & & $\begin{array}{c}\text { Fire lit for } \\
\text { game or } \\
\text { divertissemen } \\
t\end{array}$ & \\
\hline $\begin{array}{c}29 \\
\text { NUTS3 } \\
\text { Central } \\
\text { South }\end{array}$ & $\begin{array}{l}\text { Creation or } \\
\text { renewal of } \\
\text { pastures }\end{array}$ & $\begin{array}{c}\text { Agricultural } \\
\text { works } \\
\text { (stubble } \\
\text { burning) }\end{array}$ & $\begin{array}{l}\text { Agricultural } \\
\text { land cleaning } \\
\text { after } \\
\text { harvesting }\end{array}$ & $\begin{array}{c}\text { Agricultural } \\
\text { land cleaning } \\
\text { after } \\
\text { harvesting }\end{array}$ & $\begin{array}{c}\text { Hunting } \\
\text { conflicts and } \\
\text { poaching }\end{array}$ & $\begin{array}{c}\text { Mental } \\
\text { troubles and } \\
\text { pyromania }\end{array}$ & $\begin{array}{c}\text { Marciano } \\
\text { et al. } \\
2010\end{array}$ \\
\hline \multirow{3}{*}{$\begin{array}{c}\text { NUTS } 2 \\
\text { Region } \\
\text { of Apulia }\end{array}$} & \multirow[t]{3}{*}{$\begin{array}{l}\text { Burning of } \\
\text { fallow land }\end{array}$} & \multirow{3}{*}{$\begin{array}{c}\text { Agricultural } \\
\text { works } \\
\text { (stubble } \\
\text { burning) }\end{array}$} & \multirow[t]{3}{*}{$\begin{array}{l}\text { Burning of } \\
\text { fallow land }\end{array}$} & $\begin{array}{l}\text { Burning of } \\
\text { rests }\end{array}$ & \multirow{3}{*}{$\begin{array}{c}\text { Creation or } \\
\text { renewal of } \\
\text { pastures }\end{array}$} & \multirow{3}{*}{$\begin{array}{c}\text { Mental } \\
\text { troubles and } \\
\text { pyromania }\end{array}$} & \multirow{3}{*}{$\begin{array}{c}\text { Regione } \\
\text { Puglia } \\
2012\end{array}$} \\
\hline & & & & $\begin{array}{c}\text { Agricultural } \\
\text { works } \\
\text { (stubble } \\
\text { burning) }\end{array}$ & & & \\
\hline & & & & $\begin{array}{l}\text { Cleaning of } \\
\mathrm{road} / \mathrm{rail} \text { road }\end{array}$ & & & \\
\hline $\begin{array}{l}\text { NUTS2 } \\
\text { Region } \\
\text { of } \\
\text { Sardinia }\end{array}$ & $\begin{array}{c}\text { Agricultura } \\
1 \text { and forest } \\
\text { activities } \\
\text { (burning of } \\
\text { stubble and } \\
\text { agricultural } \\
\text { wastes) }\end{array}$ & Rekindle & $\begin{array}{l}\text { Careless use } \\
\text { of machinery }\end{array}$ & $\begin{array}{c}\text { Non- } \\
\text { compliance } \\
\text { with } \\
\text { Regional } \\
\text { regulations }\end{array}$ & N.A & N.A & $\begin{array}{l}\text { Lovregli } \\
\text { o et al. } \\
2014\end{array}$ \\
\hline
\end{tabular}

difference of experts' perspective. The main negligent reasons (classified in rank 1) in all the studyareas were agricultural burnings (e.g. stubble burning, creation or renewal of pastures, burning of fallow, burning of rests, agricultural land cleaning after harvesting). Also considered in the rank of the six most important fire outbreak motives appear: fire-crackers and bottle-rockets, careless use of 
machinery, cleaning of road/railroad, hot vehicle exhaust pipes, cigarettes remains, and illegal garbage dumping and burning. Garbage dumping and burning is a negligent action in the current EU causes classification (Camia et al. 2013), where it is considered a form of waste management. Anyhow, mainly in the South of Italy, it is related to illegal dumping and successive burning by organized criminality, for which illegal waste and garbage traffic and disposal represent an important ecobusiness; for this it must be classified as voluntary, related to illegal profits.

Deliberate fires motives in the Italian case-studies appeared very diverse and with different profiles. The most important motives are profit activities to obtain goods, job or even money (e.g. hunting products in areas scorched by fire passage as mushrooms and wild asparagus; building speculation; vegetation burning to earn agricultural land; fire lit to depreciate tourist area; fire caused with the intent of being included in fire-fighting crews). Social and interpersonal tensions (e.g. hunting conflicts and poaching, ownership controversies) can also explain some fire setting. Some forest fires events could be attributed to negligent behaviours (e.g. fire-crackers and bottle-rockets; careless use of machinery; hot vehicle exhaust pipes). The experts also identified fire outbreaks as a mean of protest and contestation against public or installed powers (e.g. retaliation against public administration and protest of seasonal fire-fighters) as well as noncompliance with regional regulations. Antisocial behaviours (e.g. pyromania, vandalism) did not appear as relevant as happened in Portugal. Rekindle was not considered significant in Italy, and was just mentioned in Sardinia Region.

Forest fires outbreaks motives hotspots in all the case-studies were related with the fire use in agriculture burnings and vegetation management. The representativeness and the profile of deliberate fire motives are distinctive between the case-studies.

\section{Discussion}

Forest fire outbreaks motives are not a fixed and immutable concept being used through the centuries. "Fire's definition has changed with its cultural circumstances. It takes its character from its context" (Pyne 2006: p.1). Main motives behind fire outbreaks found in the study-areas can be categorized in three main groups: traditional fire use (TFU) which refers to communities using fire for land and resources management purposes, based on traditional know-how (Lázaro 2010); deliberate fires; and rekindle, each of them putting different challenges in developing measures to reduce their incidence. TFU explains most of the fires in Portugal, Italy and Algeria as it is a deeply-rooted tool for land and resources management purposes, namely agricultural or forest residues burning and for creating or renovating pastures. TFU has been facing a very restrictive legal framework (Lovreglio and Leone 2010) resulting from the incapacity to understand that "fire for humanity is more than a problem or a process. It is a relationship" (Pyne 2006: p.6). The illegal use of fire is often carried out as a hit-andrun practice (e.g. by shepherds setting fires during the night and escaping to avoid prosecution) many times taking place even in days of high fire danger which can provoke the occurrence of large and severe fires. TFU still represents an efficacious way of "problems-solving" by aged rural societies, whose members persist with practices now considered with disdain by the dominant urban society; the latter sometimes adopts a top-down approach deciding plans and programs in the rural context without concertation nor listening the rural communities. For decades state agencies ignored this reality and the criminalization of TFU in land management activities and the lack or absence of alternatives just aggravated the problem. Problems solving through the use of fire fits with the relevant concept of " $m a n$ responsible, but not guilty" as theorized by Dumas et al.(2013) and well indicates a possible direction for prevention measures based on the scientific knowledge, respect and wise re-utilization of T.E.K. (Traditional Ecologic Knowledge: Ribet 2002) in rural areas. The criminalization of TFU and the public strategies to deal with high impact fire events, mainly through subsidies to overlap property damages, can inhibit the TFU and transform it in deliberate fire. Considering the high representativeness of the negligent use of fire in agricultural and forestry works in the investigated fires with known causes (46\% in 2012 in Portugal, according to ICNF 2012), an improved action on 
TFU could have a significant contribution for a sustained reduction of fire occurrence. The concept of integrated fire management offers the framework to enhance TFU (FAO 2006; Silva et al. 2010) which implies making communities part of the solution requiring a community-based fire management (CBFiM) approach i.e. "an approach to fire management in which local communities are actively engaged in the development, and in some instances the implementation, of fire management strategies designed to prevent, control or utilize fires in ways that will improve their livelihood, health and security" (FAO 2011: p.4).

Deliberate fires in all study areas represented only a part of the arson motive classification as proposed by NCAVC (National Centre for the Analysis of Violent Crime) of FBI Academy (Icove and Estepp 1987; Douglas et al. 1992), namely: vandalism, excitement, revenge, crime concealment, profit, and extremist. These classes were also proposed by the new classification scheme of fire causes in EU (Camia et al. 2013). In the study-areas the classes retrieved were mainly vandalism, revenge, and profit (including some cases of deliberate fires by organized crime, e.g. fire lit to depreciate tourist area as was mentioned in Italy).

"Logics" behind deliberate fires can be a consequence of processes acting: (i) at different spatial scales (e.g. some fires are related with local conflicts that originate revenges; others can be associated with the constraints of a top-down approach by state agencies, interest of non-local groups or even criminal organizations); (ii) with different frequency (i.e. some fires are isolated events; others can have a high frequency reflecting the repetition of the same fire outbreak motive). The representativeness and the profile of deliberate fire outbreak motives resulted distinctive between the case-studies. Most of the motives identified in the case-studies appeared as a consequence of local dynamics reinforcing the importance of adopting CBFiM approach with adapted strategies of intervention. A small part of deliberate fires can be interpreted as a reaction from the peasantry to the exclusion from their environment imposed by the urban society (Zarraga Moreno 1988) and the top down approach used by the state supporting policies that affect the forms of local life (e.g. forestation of commons, too restrictive nature conservation policies, precluding hunting ). Others can be related with taking profit of sectorial policies (e.g. hunting law, nature conservation law) or spatial planning (e.g. urban sprawl planning). Others can result from the management of resources imposed without assessing the impacts on the economic dynamic, environment quality and social relationships in each territory. In some cases they can represent a reaction against benefits established by law which can create conditions favourable to the exploitation of local resources by people from outside the communities, for instance changes of land use. Antisocial behaviours in Algeria and Italy (e.g. pyromania, vandalism) did not appear as important as happened in Portugal where pyromania can be related with the misuse of the term. There are different solutions to deal with deliberate fires. Law enforcement is important not only to penalize behaviours but mainly to control business opportunities related with fires. However it is not the only solution mainly because "a one fits all" strategy more than solving a problem can develop it.

The importance of rekindle is different among the case-studies considered in this paper. The frequency of fire restart is higher in Portugal and the situation has aggravated over the years (Pacheco et al. 2012). "The high number of rekindles results from the pressure on the suppression system which works at constantly very high levels of capacity utilization, and is constantly requested to immediately combat all the new fires" (Pacheco et al. op. cit.: p.10). Rekindle is important in Algeria too and can be explained by the pressure on the suppression apparatus, adding to the specific lack of security in some forested areas due to problems of terrorism, which make dangerous working in forests (Meddour-Sahar et al. 2013). In Italy, rekindle is not present in the official list of motives, with the exception of the autonomous Region of Sardinia where it appeared in the second rank. Reducing the incidence of rekindle could be achieved directly by enhancing suppression effectiveness (e.g. better training in mop-up operations), active surveillance, increased crew capacity and by adjusting dispatch rules (Pacheco et al. 2014). Indirectly, it could result from the reduction of the number of fire outbreaks which implies a decrease of the pressure on suppression apparatus. Pacheco et al. (2013) presented 
quantitative evidences of the advantages of investing in prevention to reduce rekindle as well as "to invert the vicious circle of more fire, more teams" (p.53).

\section{Conclusion}

It is a common place to say that addressing current forest fire problems requires more balanced suppression and prevention policies. The "firefighting trap" pointed out for the need to increase fire prevention clearly identifying the pitfalls of a single suppression approach. However, it is not possible to enhance prevention without a deep knowledge on the complexity of fire causes and outbreaks motives. This study showed the importance of adopting a cause-based approach focusing on the underlying reasons that induce specific negligent or deliberate behaviours.

Fire occurrence cannot be understood if disconnected from the social environment in which is produced (Zarraga Moreno 1988). The theoretical knowledge of the cultural and social construction of fire outbreak motives based on empirical studies is one of the requirements of paramount importance to enhance prevention. Without this scientific knowledge and its integration with local knowledge and the interests of the communities, the development of prevention programs can be misleading and contribute to discrediting the indisputable role of prevention. However, the immediate consequence should not be the design of a general model indistinctly applied in all contexts even with similar characteristics. From an operational point of view the theoretical framework should support prevention plans using a place-based approach, taking into account that more superficial is the knowledge of forest fire outbreak motives more inefficient and dangerous can be the transfer of findings from one context to another even with the same profile. An improved knowledge on fire ignitions motives can enhance forest fire prevention as implies a collaborative effort and communication between state agencies and local communities, thus necessarily adopting a CBFiM approach. However, it does not mean "a "one size fits all" approach, but rather it must be tailored to meet specific needs and circumstances to be an effective and sustainable approach to fire management" (FAO 2011: p.1-2).

Fire prevention oriented to the reduction of fire outbreaks is a complex issue comprising three processes: (i) knowledge of man-caused ignition motives; (ii) the development of specific targeted awareness campaigns promoting communities' engagement to change attitudes and behaviours; (iii) and concertation between communities and public organizations. The latter is a very complex and ambitious goal. It needs to accommodate several interests and solve conflicts between several actors (e.g. forest services, shepherds, farmers) through proper communication, finding concerted and socially acceptable solutions, defining new relations between public policies, administration, and communities.

\section{Acknowledgment}

The first author would like to thank the experts from the North Portugal municipalities for their collaboration in this research and CEGOT - Centre for Geography Studies and Spatial Planning, a research unit funded by FCT- Portuguese Foundation for Science and Technology, (PESTOE/SADG/UI4084/2014) for funding the participation in the VII International Conference in Forest Fire Research.

\section{References}

APAS, IDEM (2003) 'Estudio Sociológico sobre la Percepción de la población española hacia los incendios forestales.' Asociación para la Promoción de Actividades Socioculturales: (MMA: Madrid). Available at http://www. idem21.com/descargas/pdfs/ IncediosForestales.pdf APAS, IDEM (2004) 'Estado del conocimiento sobre las causas de los incendios forestales en España.' (MMA: Madrid) 
Ben Jamaa ML, Belhaj S (2011) Forest Fire in Tunisia: importance and prevention. CypFire 23-2 Cypress and forest fires: a practical manual. Reports of the Training School. (Florence, IT). Available at http://cupressus.ipp.cnr.it/cypfire/files/Brochure\%20Summer\%20School-5.pdf

CFS (2013)'Incendi boschivi. Dati di sintesi 2012.' (Corpo Forestale dello Stato: Roma)

Camia A, Durrant T, San-Miguel-Ayanz J (2013) 'Harmonized classification scheme of fire causes in the EU adopted for the European Fire Database of EFFIS Executive report.'(JRC, European Commission: Ispra)

Collins RD (2012) 'Forest Fire Management in Portugal: Developing System Insights through Models of Social and Physical Dynamics' (SM thesis). (Massachusetts Institute of Technology: Massachusetts)

Collins RD, de Neufville R, Claro J, Oliveira T, Pacheco AB (2013) Forest fire management to avoid unintended consequences: A case study of Portugal using system dynamics. Journal of Environmental Management 130, 1-9.

De Las Heras J, Salvatore R, Rodrigues MJ, Lovreglio R, Leone V, Giaquinto P, Notarnicola A (2007) Wildfire motivation survey through the Delphi method. Actas de la IV Conferencia Internacional sobre Incendios Forestales. (Sevilla: SP). Available at http://www.fire.unifreiburg.de/sevill2007/contributions/doc/SESIONES_TEMATICAS/ST4/Hera s_et-AL_SPAIN_ITALY.pdf

Dolz Reus ML, Franco Irastorza I (2005) State of the art of forest fire causes in Spain. In 'Proceedings of II International Conference on Prevention Strategies of Fires in Southern Europe' (Ed Centre Tecnologic Forestal de Cataluña) pp.325-331. (Centre Tecnologic Forestal de Cataluña: Solsona)

Douglas E, Burgess AW, Burgess AG, Ressler RK (1992) 'Crime Classification Manual: A Standard System for Investigating and Classifying Violent Crimes.' (Lexington Books: New York)

Dumas P, Toussaint M, Herrenschmidt J-B, Conte A, Mangeas M (2013) Le risque de feux de brousse sur la Grande Terre de Nouvelle-Calédonie: l'Homme responsable, mais pas Coupable. Revue Géographique de l'Est [En ligne] 53/ 1-2 |. Available at http://rge.revues.org/4598

EFIMED (2012) The fight against forest fires: an inconvenient truth? EFIMED network newsaugust2012, 1. Available at http://news.efi.int/newsletter/view/efimed-newsletter-august-2012/1044

European Commission (2013) 'Forest Fires in Europe, Middle East and North Africa.2012.' (Publications Office of the European Union: Luxembourg)

FAO (1999) 'FAO Meeting on Public Policies Affecting Forest Fires.' (FAO: Rome)

FAO (2006) 'Fire Management Voluntary Guidelines. Fire Management.' (FAO: Rome)

FAO (2011) 'Community-based fire management: A review.' (FAO: Rome)

FAO (2012) 'State of Mediterranean Forests (SoMF) Concept Paper.' (FAO: Rome)

Franco Irastorza I, Dolz Reus ML (2007) Análisis de la percepción de la sociedad ante el problema de los incendios forestales: metodología y resultados. In 'Actas de la IV Conferencia Internacional sobre Incendios Forestales". (Sevilla: SP). Available at http://www.fire.uni-freiburg.de/sevilla2007/contributions/doc/cd/SESIONES_TEMATICAS/ST1/Franco_Dolz_SPAIN.pdf

ICNF (2012) 'Relatório anual de áreas ardidas e incêndios florestais em Portugal Continental'. (ICNF, ANPC: Lisboa)

INE (2012) 'CENSOS 2011. RESULTADOS DEFINITIVOS, Norte'' (INE: Lisboa). Available at http://www.ine.pt/xportal/xmain?xpid=INE\&xpgid=ine_main

Icove DJ, Estepp MH (1987) Motive-Based Offender Profiles of Arson and Fire Related Crimes. FBI Law Enforcement Bulletin 56, 17-23.

Lázaro A (2010) Development of prescribed burning and suppression fire in Europe. In 'Best practices of fire use - Prescribed burning and suppression in selected case-study regions in Europe'. (Eds C Montiel, D Kraus) pp. 17-31. (European Forest Institute: Joensuu)

Leone V, Lovreglio R (2009) Parco Nazionale del Gargano Piano di Previsione, Prevenzione e lotta attiva contro gli incendi boschivi Primo Aggiornamento 2009-2011. Available at http://www.minambiente.it/pagina/parco-nazionale-del-gargano-0\#sthash.waDv9yTY.dpuf 
Leone V, Lovreglio R (2003) Human fire causes: a challenge for modelling. In 'Innovative Concepts and Methods in Fire Danger Estimation’. (Eds E Chuvieco, P Martín, C. Justice) pp.89-98. (Ghent University - EARSeL: Ghent)

Leone V, Lovreglio R, Pilar Martín M, Martínez J, Vilar L (2009) Human Factors of Fire Occurrence in the Mediterranean. In 'Earth Observation of Wildland Fires in Mediterranean Ecosystems'. (Ed E. Chuvieco) pp.149-170. (Springer-Verlag: Berlin, Heidelberg)

Lovreglio R, Leone V, Giaquinto P, Notarnicola A (2006) New tools for the analysis of fire causes and their motivations: the Delphi technique. Forest Ecology and Management 234, 18-33.

Lovreglio R, Rodrigues MJ, Silletti G, Leone V (2008) Applicazione del metodo Delphi per l'analisi delle motivazioni degli incendi: il caso Taranto. L'Italia Forestale e Montana 5, 427-447.

Lovreglio R, Leone V, Giaquinto P, Notarnicola A (2010a) Wildfire cause analysis: four case-studies in southern Italy. iForest 3, 8-15.

Lovreglio R, Rodrigues MJ, Notarnicola A, Leone V (2010b) From fire motives survey to prevention: the case of Cilento and Vallo di Diano National Park (Italy). In 'Proceedings of the VI International Conference on Forest Fire Research'. (Ed DX Viegas) pp.1-6 (Paper 18) (ADAI/CEIF, University of Coimbra: Coimbra)

Lovreglio R, Mou G, Leone V (2014) Forest fire motives in Sardinia through the perception of experts. In 'Advances in Forest Fire Research' (Ed DX Viegas). (Coimbra University Press: Coimbra) (in press)

Marciano A, Lovreglio R, Patrone A, Notarnicola A, Leone V (2010) Tecniche di analisi delle motivazioni degli incendi. Applicazione nel territorio del Parco Nazionale del Cilento e Vallo di Diano. Sherwood 162, 13-18.

Marino E, Hernando C, Planelles R, Madrigal J, Guijarro M, Sebastian A (2014) Forest fuel management for wildfire prevention in Spain: a quantitative SWOT analysis. International Journal of Wildland Fire 23, 373-384.

McCaffrey S (2004) Thinking of Wildfire as a Natural Hazard. Society and Natural Resources 17, 509-516.

Meddour-Sahar O, Meddour R, Leone V, Lovreglio R, Derridj A (2013) Analysis of forest fires causes and their motivations in North Algeria: the Delphi technique. iForest-Biogeosciences and Forestry 6, 247-254.

Meddour-Sahar O (2014) 'Les feux de forêt en Algérie: Analyse du risque, étude des causes, évaluation du dispositif de défense et des politiques de gestion.' (Université Mouloud Mammeri: Tizi Ouzou)

Molina D, Castellnou M, García-Marco D, Salgueiro A (2010) Improving fire management success through fire behaviour specialists. In 'Towards Integrated Fire Management-Outcomes of the European Project Fire Paradox’. (Eds JS Silva, F Rego, P Fernandes, E Rigolot) pp. 105-119. (European Forest Institute: Joensuu)

Montiel C, Herrero G (2010) An overview of policies and practices related to fire ignitions at the European Union level. In 'Towards Integrated Fire Management-Outcomes of the European Project Fire Paradox’. (Eds JS Silva, F Rego, P Fernandes, E Rigolot) pp. 35-46. (European Forest Institute: Joensuu)

Pacheco AP, Claro J, Oliveira T (2012) Rekindle dynamics: validating the pressure on wildland fire suppression resources and implications for fire management in Portugal. In 'Modeling, monitoring and management of forest fires III'. (Ed Wessex Institute of Technology). (WIT-Press: Ashurst, Southampton)

Pacheco AP, Claro J, Oliveira T (2014) Simulation analysis of the impacts of ignitions, rekindles, and false alarms on forest fires suppression. Canadian Journal of Forest Research 44, 45-55.

Pyne S (2006) Fire Ecology: Issues, Management, Policy, and Opinions. A forum for the association for fire Ecology. Fire Ecology 2, 1-6.

Raftoyannis Y, Nocentini S, Marchi E, Calama Sainz R, Garcia Guemes C, Pilas I, Peric S, Paulo JA, Moreira-Marcelino AC, Costa-Ferreira M, Kakouris E, Lindner M (2014) Perceptions of forest 
experts on climate change and fire management in European Mediterranean forests. iForestBiogeosciences and Forestry 7, 33-41.

Regione Puglia (2012) Piano di prevenzione, prevenzione e lotta attività contro gli incendi boschivi 2012-2014. Legge 353/2000. Bollettino Ufficiale Regione Puglia, 59, 12455-13042.Available at http://www.protezionecivile.puglia.it/public/news.php?extend.630.10

Ribet N (2002) La maîtrise du feu un travail "en creux" pour façonner les paysages. In "Travail et paysages'. (Ed D. Woronoff) pp.167-198. (Éditions du CTHS: Paris). Available at http://www.ecoanthropologie.cnrs.fr/pdf/Ribet_Trav-Paysa.pdf

Silva JS, Rego F, Fernandes P, Rigolot E (Eds) (2010) 'Towards Integrated Fire Management Outcomes of the European Project Fire Paradox.' (European Forest Institute: Joensu)

Sebastián-López A, Carmen Hernando L, Planelles R, Buffoni A, Boström C, Alves R, Jappiot M, San Miguel Ayanz J (2011) EU-FIRESMART: Forest and land management options to prevent unwanted forest fires. '5th International Wildland Fire Conference', 9-13 May. (South Africa)

Wrathall MA (2005) Motives, Reasons, and Causes. In ' The Cambridge Companion to MerleauPonty'. (Eds T Carman, MBN Hansen) pp. 111-128. (Cambridge University Press: Cambridge)

Xanthopoulos G (2012) Evolution of the forest fire problems in Greece and mitigation measures for the future. In 'Proceedings of the $1^{\text {st }}$ International Conference in Safety and Crisis Management in the Construction Tourism and SME Sectors' (Eds G Boustras, N Boukas) pp. 736-747 (Brown Walker Press: Boca Raton)

Zarraga Moreno JL (1988) Los incendios forestales y las actitudes de la población de las comarcas afectadas. (Servicio de Estudios e Informes del I.A.R.A. I.A.R.A.: Sevilla) 